PROCEEDINGS OF THE

AMERICAN MATHEMATICAL SOCIETY

Volume 88, Number 2, June 1983

\title{
A GENERALIZATION OF THE POINCARÉ-BIRKHOFF THEOREM
}

\author{
WEI-YUE DING
}

\begin{abstract}
A generalized form of the Poincaré-Birkhoff theorem is proved. The generalization is useful for the further applications of this famous fixed point theorem.
\end{abstract}

1. Introduction and statement of the result. "To establish the existence of periodic orbits in the restricted problem of three bodies Poincare introduced his celebrated 'last geometric theorem' but was unable to prove it... A number of mathematicians had attempted to prove this theorem and at least one erroneous proof was published. Urged on by his colleagues, Birkhoff gave a beautiful proof of the theorem that went directly to the heart of the problem. Modified forms of the theorem were also presented by Birkhoff."-quoted from Marston Morse [3].

Today, this Poincaré's last geometric theorem is known as the Poincaré-Birkhoff theorem. In the following, we will give a statement of this theorem in modern terms.

Let $\mathbf{R}^{2}$ be the $(x, y)$-plane. Let $H: r>0$ be the half-plane of the $(r, \theta)$-plane. We will regard $H$ as a covering space of $\mathbf{R}^{2} \backslash\{O\}$, where $O$ is the origin of $\mathbf{R}^{2}$. Let $P: H \rightarrow \mathbf{R}^{2} \backslash\{O\}$ be the usual covering projection: $P(r, \theta)=(r \cos \theta, r \sin \theta)$. Recall that, for any continuous map $F: D \subset \mathbf{R}^{2} \backslash\{O\} \rightarrow \mathbf{R}^{2} \backslash\{O\}$, a map $\tilde{F}: P^{-1}(D) \rightarrow H$ is called a lifting of $F$ to $H$, if the following relation holds:

$$
P \circ \tilde{F}=F \circ P \text {. }
$$

Denote by $A_{0}$ the annulus: $R_{1}^{2} \leq x^{2}+y^{2} \leq R_{2}^{2}, 0<R_{1}<R_{2}$.

POINCARÉ-BIRKHOFF THEOREM. Let $T$ be an area-preserving homeomorphism of $A_{0}$ onto itself such that both boundary circles of $A_{0}$ are invariant under $T$. Suppose that $T$ has a lifting $\tilde{T}: P^{-1}\left(A_{0}\right) \rightarrow P^{-1}\left(A_{0}\right)$ defined by

$$
r^{*}=f(r, \theta), \quad \theta^{*}=\theta+g(r, \theta)
$$

where $f$ and $g$ are continuous and $2 \pi$-periodic in $\theta$. If the "twist condition": $g\left(R_{1}, \theta\right) g\left(R_{2}, \theta\right)<0$ holds, then $\tilde{T}$ has at least two fixed points such that their images under the projection $P$ are two different fixed points of $T$.

In this theorem, the condition that $T$ maps $A_{0}$ onto itself is a severe restriction for the application of the theorem. This was certainly noticed by Birkhoff who was interested in the dynamical applications of the theorem. He then made efforts to remove this condition. In [2], he succeeded to such an extent that the outer boundary circle of $A_{0}$ is permitted to vary under $T$ while the inner boundary circle remains invariant. This result now can be formulated in a more general form through a modification by H. Jacobowitz (see [4]).

Received by the editors February 25, 1981

1980 Mathematics Subject Classification. Primary 54H25.

(c) 1983 American Mathematical Society 0002-9939/81/0000-0449/\$02.00 
Let $A_{1}$ denote an annular region in $\mathbf{R}^{2}$ such that its inner boundary curve $C_{1}$ is a circle: $x^{2}+y^{2}=R_{1}^{2}, R_{1} \geq 0$, and the outer boundary curve $C_{2}$ is a closed simple curve. Note that $C_{1}$ will degenerate into the origin in the case $R_{1}=0$. In this degenerate case we assume that $A_{1}$ does not contain the origin.

Modified PoinCARÉ-Birkhoff Theorem. Let $T: A_{1} \rightarrow T\left(A_{1}\right) \subset \mathbf{R}^{2} \backslash\{O\}$ be an area-preserving homeomorphism. Suppose that the boundary circle $C_{1}$ is invariant under $T$. (In the degenerate case this means the origin is also the inner boundary of $T\left(A_{1}\right)$.) Moreover, suppose that $T$ has a lifting $\tilde{T}$ defined by (1.1) such that $g(r, \theta)<0$ on $P^{-1}\left(C_{2}\right)$ and $\lim _{r \rightarrow R_{1}}$ inf $g(r, \theta)>0$. Then $\tilde{T}$ has at least two fixed points such that their images under $P$ are two different fixed points of $T$.

One asks naturally: what will happen if we allow the inner boundary also variant under $T$ ? This problem is of importance because often the map $T$ raised in a concrete problem does not possess the obvious invariant curve or fixed point. It is the aim of this paper to answer the above question. Our result is the following theorem.

Let $A$ denote an annular region. The inner boundary $C_{1}$ and the outer boundary $C_{2}$ of $A$ are two closed simple curves. By $D_{i}$ we denote the open region bounded by $C_{i}, i=1,2$.

THEOREM 1. Let $T: A \rightarrow T(A) \subset \mathbf{R}^{2} \backslash\{O\}$ be an area-preserving homeomorphism. Suppose that

(1) the inner boundary curve $C_{1}$ is star shaped about the origin;

(2) $T$ has a lifting $\tilde{T}$ defined by $(1.1)$ such that $g(r, \theta)>0$ on $P^{-1}\left(C_{1}\right)$ and $g(r, \theta)<0$ on $P^{-1}\left(C_{2}\right)$;

(3) there exists an area-preserving homeomorphism $T_{0}: \bar{D}_{2} \rightarrow \mathbf{R}^{2}$, which satisfies $T_{0} \mid A=T$ and $O \in T_{0}\left(D_{1}\right)$.

Then $\tilde{T}$ has at least two fixed points such that their images under $P$ are two different fixed points of $T$.

We have proved this theorem for the case that $A$ is the standard annulus $A_{0}: R_{1}^{2} \leq$ $x^{2}+y^{2} \leq R_{2}^{2}$, in a previous paper [6]. The present more general form is necessary for certain applications, as is shown in [5]. The condition (1) of the theorem is crucial for our proof. However, we doubt its necessity for the theorem. As to the condition (3), its necessity can be seen from the following simple example.

Let $T$ be a mapping from the annulus $2^{-2} \leq x^{2}+y^{2} \leq 2^{2}$ into $\mathbf{R}^{2} \backslash\{O\}$ such that $T$ has a lifting defined by $r^{*}=\sqrt{r^{2}+1}, \theta^{*}=\theta+1-r$. It is clear that $T$ is uniquely determined by $\tilde{T}$. Furthermore, $T$ is a homeomorphism which preserves the element of area $d x d y=r d r d \theta$. We have $g(r, \theta)=1-r$ in this example, which is positive on $r=\frac{1}{2}$ and negative on $r=2$. Therefore, all assumptions except (3) of Theorem 1 are satisfied. However, $\tilde{T}$ has no fixed point since $r^{*}>r$ for any $r$.

2. Proof of Theorem 1. The idea of our proof is very simple. We want to construct a new mapping $T_{2}: \bar{D}_{2} \backslash\{O\} \rightarrow \mathbf{R}^{2} \backslash\{O\}$ which possesses the following properties:

(a) $T_{2}$ is an area-preserving homeomorphism.

(b) The restriction of $T_{2}$ on $A$ agrees with $T$, i.e. $T_{2} \mid A=T$.

(c) The origin is the inner boundary of $T_{2}\left(\bar{D}_{2} \backslash\{O\}\right)$. 
(d) $T_{2}$ has a lifting $\tilde{T}_{2}$ defined by

$$
r^{*}=f_{2}(r, \theta), \quad \theta^{*}=\theta+g_{2}(r, \theta),
$$

such that $f_{2}=f, g_{2}=g$ on $P^{-1}(A)$, i.e. $\tilde{T}_{2} \mid P^{-1}(A)=\tilde{T}$ and $g_{2}(r, \theta) \geq a>0$ on $P^{-1}\left(D_{1} \backslash\{O\}\right)$.

If such $T_{2}$ exists, we see from (a), (c), (d) and (2) of Theorem 1 that $T_{2}$ and its lifting $\tilde{T}_{2}$ satisfy all assumptions of the Modified Poincaré-Birkhoff Theorem. It follows that $\tilde{T}_{2}$ has at least two fixed points such that their images under $P$ are two different fixed points of $T_{2}$. However, $\tilde{T}_{2}$ cannot have any fixed point in $P^{-1}\left(D_{1} \backslash\{O\}\right)$, since $g_{2}(r, \theta)>0$ on this region by (d). Therefore, all fixed points of $\tilde{T}_{2}$ must be contained in $P^{-1}(A)$. As $\tilde{T}_{2} \mid P^{-1}(A)=\tilde{T}$ and $T_{2} \mid A=T$, we conclude at once that $\tilde{T}$ has at least two fixed points such that their images under $P$ are two different fixed points of $T$, thus completing the proof.

We now proceed to construct the mapping $T_{2}$.

LEMMA 1. There exists an area-preserving homeomorphism $T_{1}: \bar{D}_{2} \rightarrow \mathbf{R}^{2}$ such that $T_{1} \mid A=T$ and $T_{1}(O)=O$.

Proof. Let $T_{0}$ be the mapping given by (3) of the theorem. Then we have $p=T_{0}^{-1}(O) \in D_{1}$. Choose points $O=P_{0}, P_{1}, P_{2}, \ldots, P_{n}=P$ in the segment $\overline{O P}$, such that the length $2 d_{i}$ of the subsegment $\overline{P_{i-1} P_{i}}(i=1,2, \ldots, n)$ is less than $d=$ $\operatorname{distant}\left(\overline{O P}, C_{1}\right)$. By (1) of the theorem, we have $d>0$. For each $i \in\{1,2, \ldots, n\}$ we construct an area-preserving homeomorphism $S_{i}$ as follows. Let $O_{i}$ denote the middle point of $\overline{P_{i-1} P_{i}}$. Choose any polar coordinate system $\left(\rho_{i}, \varphi_{i}\right)$ in $\mathbf{R}^{2}$ which has $O_{i}$ as its pole. With this coordinate system define $S_{i}: \mathbf{R}^{2} \rightarrow \mathbf{R}^{2}$ by

$$
\rho_{i}^{*}=\rho_{i}, \quad \varphi_{i}^{*}=\varphi_{i}+h_{i}\left(\rho_{i}\right) .
$$

Here, $h_{i}\left(\rho_{i}\right)$ is a $C^{\infty}$ function satisfying that $h_{i}\left(\rho_{i}\right)=0$ for $\rho_{i} \geq \frac{1}{2}\left(d_{i}+d\right), h_{i}\left(\rho_{i}\right)=\pi$ for $0<\rho_{i} \leq d_{i}$. It is clear from this definition that $S_{i} \mid A=$ identity and $S_{i}\left(P_{i-1}\right)=$ $P_{i}$. It is also easy to check that $S_{i}$ is an area-preserving diffeomorphism. Now, let $S=S_{n} \circ S_{n=1} \circ \cdots \circ S_{1}$. Then $S$ is an area-preserving diffeomorphism satisfying that $S \mid A=$ identity and $S(O)=P$. Finally, define $T_{1}=T_{0} \circ S$. We see that $T_{1}$ is an areapreserving homeomorphism since it is a composition of such mappings. Moreover, $T_{1}\left|A=T_{0} \circ S\right| A=T_{0} \mid A=T$, and $T_{1}(O)=T_{0}(S(O))=T_{0}(P)=T_{0}\left(T_{0}^{-1}(O)\right)=O$. This completes the proof of Lemma 1. by

Now, let $\tilde{T}_{1}$ be a lifting of the restriction of $T_{1}$ on $\bar{D}_{2} \backslash\{O\}$. Suppose $\tilde{T}_{1}$ is defined

$$
r^{*}=f_{1}(r, \theta), \quad \theta^{*}=\theta+g_{1}(r, \theta) .
$$

Since $T_{1} \mid A=T$, we can choose $\tilde{T}_{1}$ such that

$$
f_{1}(r, \theta)=f(r, \theta), \quad g_{1}(r, \theta)=g(r, \theta) \quad \text { on } P^{-1}(A),
$$

i.e. $\tilde{T}_{1} \mid P^{-1}(A)=\tilde{T}$. By (2) of Theorem 1 , we have $g_{1}=g>0$ on $P^{-1}\left(C_{1}\right)$. Since $g_{1}(r, \theta)$ is $2 \pi$-periodic in $\theta$ there exists a neighborhood $U$ of $C_{1}$ such that

$$
g_{1}(r, \theta) \geq a>0 \text { on } P^{-1}(U),
$$

for some constant $a$. Let $C_{1}^{\prime}$ be any smooth closed simple curve in $\bar{D}_{1} \cap U$ which is strictly star shaped about the origin. By strictly star shaped we mean that any ray from the origin hits $C_{1}^{\prime}$ at just one point and nontangentially. The existence of such curves is guaranteed by (1) of the theorem. Let $(x, y)$ be any point in $\mathbf{R}^{2} \backslash\{O\}$. The 
ray starting from the origin and passing $(x, y)$ will hit $C_{1}^{\prime}$ at a unique point $P(x, y)$. Define a function $\alpha: \mathbf{R}^{2} \backslash\{O\} \rightarrow \mathbf{R}_{+}$by $\alpha(x, y)=1 /|P(x, y)|$, where $|\cdot|$ denotes the Euclidean norm of $\mathbf{R}^{2}$. Because $C_{1}^{\prime}$ is smooth and strictly star shaped the function $\alpha$ is continuously differentiable. Note that for any $k>0$ we have

$$
\alpha(k x, k y)=\alpha(x, y) \text {. }
$$

Differentiating with respect to $k$ and letting $k=1$, we get

$$
x \frac{\partial \alpha}{\partial x}+y \frac{\partial \alpha}{\partial y}=0
$$

Define $\lambda(x, y)=\alpha(x, y) \sqrt{x^{2}+y^{2}}$ for $x^{2}+y^{2} \neq 0$, and $\lambda(0,0)=0$. It is easy to see that $\lambda^{-1}(1)=C_{1}^{\prime}$, and for any $\mu>0, \lambda^{-1}(\mu)=C_{\mu}^{\prime}$ is a similar curve of $C_{1}^{\prime}$ about the origin. We can also easily see that $\lambda^{-1}[0,1)=D_{1}^{\prime}$ is just the open region bounded by $C_{1}^{\prime}$. Choose $\epsilon>0$ so small that

$$
\lambda^{-1}(1-\epsilon, 1) \subset U \text {. }
$$

For $\mu>0$, set

$$
\begin{aligned}
& \beta(\mu)=\sup \left\{x^{2}+y^{2} \mid(x, y) \in C_{\mu}^{\prime}\right\} \\
& \gamma(\mu)=\sup \left\{\left|g_{1}(r, \theta)\right| \mid(r, \theta) \in P^{-1}\left(C_{\mu}^{\prime}\right)\right\}
\end{aligned}
$$

LEMMA 2. There exists an area-preserving homeomorphism $R$ of $\mathbf{R}^{2} \backslash\{O\}$ onto $\mathbf{R}^{2} \backslash\{O\}$ such that $R$ has a lifting $\tilde{R}$ defined by

$$
r^{*}=\phi(r, \theta), \quad \theta^{*}=\theta+\psi(r, \theta)
$$

which satisfies

$$
\begin{gathered}
\phi(r, \theta)=r, \quad \psi(r, \theta)=0 \text { for }(r, \theta) \in P^{-1}\left(\mathbf{R}^{2} \backslash D_{1}^{\prime}\right), \\
\psi(r, \theta) \geq 0 \text { for all }(r, \theta) \in H, \\
\psi(r, \theta) \geq \gamma(\mu)+a \quad \text { for }(r, \theta) \in P^{-1}\left(C_{\mu}^{\prime}\right) \text { and } \mu \in(0,1-\epsilon] .
\end{gathered}
$$

Proof. Let $F:(0,+\infty) \rightarrow \mathbf{R}$ be a $C^{\infty}$ function satisfying that $F(u)=0$ for $u \geq 1, F(u)>0$ for $u \in(1-\epsilon, 1)$, and $F(u) \geq u^{-1} \beta(u)[\gamma(u)+a]$ for $u \in(0,1-\epsilon]$. Set

$$
H(x, y)=\int_{0}^{\lambda(x, y)} F(u) d u .
$$

Consider the following Hamiltonian system defined on $\mathbf{R}^{2} \backslash\{O\}$ :

$$
\left\{\begin{array}{l}
\dot{x}=-\frac{\partial H}{\partial y} \equiv-F(\lambda(x, y))\left[\frac{y \alpha(x, y)}{\sqrt{x^{2}+y^{2}}}+\frac{\partial \alpha}{\partial y} \sqrt{x^{2}+y^{2}}\right] \\
\dot{y}=\frac{\partial H}{\partial x} \equiv F(\lambda(x, y))\left[\frac{x \alpha(x, y)}{\sqrt{x^{2}+y^{2}}}+\frac{\partial \alpha}{\partial x} \sqrt{x^{2}+y^{2}}\right]
\end{array}\right.
$$

By the definition of $F(u)$ and $\lambda(x, y)$, the set $\lambda^{-1}[1, \infty)=\mathbf{R}^{2} \backslash D_{1}^{\prime}$ consists of all stationary points of the system (2.11). Also, we can easily verify that $\lambda(x, y)$ is a first integral of (2.11) and for each $\mu \in(0,1), C_{\mu}^{\prime}$ is an integral curve of (2.11). 
Let $(\bar{x}(t, x, y), \bar{y}(t, x, y))$ denote the solution of $(2.11)$ that satisfies the initial condition $\bar{x}(0)=x, \bar{y}(0)=y$. We define the mapping $R: \mathbf{R}^{2} \backslash\{O\} \rightarrow \mathbf{R}^{2} \backslash\{O\}$ by

$$
R(x, y)=(\bar{x}(1, x, y), \bar{y}(1, x, y)) .
$$

Note that $R$ is the time-one mapping of the Hamiltonian flow induced by (2.11). Therefore $R$ is an area-preserving homeomorphism, and for $(x, y) \in C_{\mu}^{\prime}, R(x, y) \in$ $C_{\mu}^{\prime}$. In particular, we have $R(x, y)=(x, y)$ for $(x, y) \in \mathbf{R}^{2} \backslash D_{1}^{\prime}$.

Now, take the transformation $x(t)=r(t) \cos \theta(t), y(t)=r(t) \sin \theta(t)$ for (2.11). The resulting equaitons for $r(t), \theta(t)$ are

$$
\left\{\begin{array}{l}
\dot{\gamma}=-\cos \theta \frac{\partial H}{\partial y}+\sin \theta \frac{\partial H}{\partial x}, \\
\dot{\theta}=\frac{1}{\gamma}\left(\cos \theta \frac{\partial H}{\partial x}+\sin \theta \frac{\partial H}{\partial y}\right) .
\end{array}\right.
$$

Employing (2.4), the second equation of (2.12) can be reduced to

$$
\dot{\theta}=\frac{\lambda(x, y) F(\lambda(x, y))}{x^{2}+y^{2}} \text {. }
$$

Let $(\bar{r}(t, r, \theta), \bar{\theta}(t, r, \theta))$ denote the solution of $(2.12)$ which satisfies $\bar{r}(0)=r, \bar{\theta}(0)=\theta$. Set $\phi(r, \theta)=\bar{\gamma}(1, r, \theta), \psi(r, \theta)=\bar{\theta}(1, r, \theta)-\theta$. Then we see that $R$ has a lifting $\tilde{R}$ which is defined by

$$
r^{*}=\phi(r, \theta), \quad \theta^{*}=\theta+\psi(r, \theta) .
$$

It is clear that $\phi(r, \theta)=r, \psi(r, \theta)=0$ on $P^{-1}\left(\mathbf{R}^{2} \backslash D_{1}^{\prime}\right)$ where every point is a stationary point of (2.12). It is also easy to see that $\lambda \circ P(r, \theta)$ is a first integral of (2.12). Thus we have

$$
\lambda \circ P[\bar{r}(t, r, \theta), \bar{\theta}(t, r, \theta)] \equiv \mu, \quad \text { for }(r, \theta) \in P^{-1}\left(C_{\mu}^{\prime}\right) .
$$

It follows from (2.13) that

$$
\dot{\bar{\theta}}(t, r, \theta)=\mu F(\mu) / \bar{r}^{2}(t, r, \theta), \quad \text { for }(r, \theta) \in P^{-1}\left(C_{\mu}^{\prime}\right) .
$$

For any $(r, \theta) \in H$, we see from (2.14) that $\dot{\bar{\theta}}(t, r, \theta) \geq 0$. Hence,

$$
\psi(r, \theta)=\bar{\theta}(1, r, \theta)-\theta \geq 0, \quad \text { for } \operatorname{each}(r, \theta) \in H .
$$

For $(r, \theta) \in P^{-1}\left(C_{\mu}^{\prime}\right)$ and $\mu \in(0,1-\epsilon],(2.14)$ and (2.6) yield

$$
\dot{\bar{\theta}}(t, r, \theta) \geq \beta(\mu)[\gamma(\mu)+a] / \bar{r}^{2}(t, r, \theta) \geq \gamma(\mu)+a .
$$

Integrating on $(0,1)$, we get

$$
\psi(r, \theta)=\bar{\theta}(1, r, \theta)-\theta \geq \gamma(\mu)+a, \quad \text { for }(r, \theta) \in P^{-1}\left(C_{\mu}^{\prime}\right), \mu \in(0,1-\epsilon] .
$$

This completes the proof of Lemma 2.

Now let $T_{1}$ and $R$ be the mappings given by Lemma 1 and Lemma 2 respectively. We define $T_{2}=T_{1} \circ R$ and verify that $T_{2}$ possesses the asserted properties (a)-(d). Since the verification of (a)-(c) is trivial, it suffices to show that (d) is satisfied by $T_{2}$.

Let $\tilde{T}_{1}$ be the lifting of $T_{1} \mid\left(\bar{D}_{2} \backslash\{O\}\right)$ defined by (2.1). Let $\tilde{R}$ be the lifting of $R$ given by Lemma 2 . Note that $\widetilde{T}_{2}=\widetilde{T}_{1} \circ \tilde{R}$ is a lifting of $T_{2}$. Suppose that $\tilde{T}_{2}$ is 
given by

By (2.1) and (2.7),

$$
r^{*}=f_{2}(r, \theta), \quad \theta^{*}=\theta+g_{2}(r, \theta) .
$$

$$
\begin{aligned}
& f_{2}(r, \theta)=f_{1}(\phi(r, \theta), \theta+\psi(r, \theta)) \\
& g_{2}(r, \theta)=\psi(r, \theta)+g_{1}(\phi(r, \theta), \theta+\psi(r, \theta)) .
\end{aligned}
$$

From (2.8) and (2.2) it follows that

$$
f_{2}(r, \theta)=f(r, \theta), \quad g_{2}(r, \theta)=g(r, \theta), \quad \text { for }(r, \theta) \in P^{-1}(A) .
$$

Suppose now $(r, \theta) \in P^{-1}\left(D_{1} \backslash\{O\}\right)$. If $(r, \theta) \in P^{-1}\left(U \backslash D_{1}^{\prime}\right)$, then

$$
g_{2}(r, \theta)=g_{1}(r, \theta) \geq a
$$

by (2.8) and (2.3). If $(r, \theta) \in P^{-1}\left(\lambda^{-1}(1-\epsilon, 1)\right) \subset P^{-1}(U)$, noting that $\tilde{R}(r, \theta) \in$ $P^{-1}\left(\lambda^{-1}(1-\epsilon, 1)\right)$ too, we have

$$
g_{2}(r, \theta) \geq g_{1}(\phi(r, \theta), \theta+\psi(r, \theta)) \geq a
$$

by (2.9) and (2.3). Finally, if $(r, \theta) \in P^{-1}\left(\lambda^{-1}(0,1-\epsilon)\right)$ then $(r, \theta) \in P^{-1}\left(C_{\mu}^{\prime}\right)$ for some $\mu \in(0,1-\epsilon)$. It follows from $(2.10)$ and $(2.6)$ that

$$
g_{2}(r, \theta) \geq \gamma(\mu)+a+g_{1} \circ \tilde{R}(r, \theta) \geq a .
$$

Here we have made use of the fact that $\tilde{R}(r, \theta) \in P^{-1}\left(C_{\mu}^{\prime}\right)$ for $(r, \theta) \in P^{-1}\left(C_{\mu}^{\prime}\right)$. In summary, we have in any case that $g_{2}(r, \theta) \geq a>0$ for $(r, \theta) \in P^{-1}\left(D_{1} \backslash\{O\}\right)$. Thus (d) is satisfied.

The proof of Theorem 1 is complete.

\section{REFERENCES}

1. G. D. Birkhoff, Proof of Poincaré's geometric theorem, Trans. Amer. Math. Soc. 14 (1913), 14-22.

2. G. D. Birkhoff, Dynamical systems, Amer. Math. Soc. Colloq. Publ., vol. 27, Amer. Math. Soc., Providence, R.I., 1927; revised 1966; reprinted 1979.

3. M. Morse, George David Birkhoff and his mathematical work, Bull. Amer. Math. Soc. 52 (1946), 357-391.

4. H. Jacobowitz, Periodic solutions of $x^{\prime \prime}+f(x, t)=0$ via the Poincaré-Birkhoff Theorem, J. Differential Equations 20 (1976), 37-52; and Corrigendum: The existence of the second fixed point: A correction to "Periodic solutions of $x^{\prime \prime}+f(x, t)=0$ via the Poincaré-Birkhoff Theorem", J. Differential Equations 25 (1977), 148-149.

5. T. R. Ding, An infinite class of periodic solutions of periodically perturbed Duffing equations at resonance, Proc. Amer. Math. Soc. 86 (1982), 47-54.

6. W. Y. Ding, Fixed points of twist mappings and periodic solutions of ordinary differential equations, Acta Math. Sinica 25, 227-235. (Chinese)

Institute of Mathematics, ACademia Sinica, Beijing, People's Republic of CHINA 55455

Current address: School of Mathematics, University of Minnesota, Minneapolis, Minnesota 